\title{
Synthesis of 2-substituted indoles and evaluation of their antibacterial activity and inhibitory effects on the efflux pump of methicillin-resistant Staphylococcus aureus
}

\author{
Suttinun Vicharn ${ }^{1}$, Jitnapa Sirirak ${ }^{1}$, Weerachai Phutdhawong ${ }^{2}$, Thongchai Taechowisan ${ }^{3}$, Waya S. Phutdhawong ${ }^{1 *}$ \\ ${ }^{1}$ Department of Chemistry, Faculty of Science, Silpakorn University, Nakhon Pathom, Thailand. \\ ${ }^{2}$ Department of Science, Faculty of Liberal Arts and Science, Kasetsart University, Kamphaeng Sean Campus, Nakhon Pathom, Thailand. \\ ${ }^{3}$ Department of Microbiology, Faculty of Science, Silpakorn University, Nakhon Pathom, Thailand.
}

\begin{tabular}{l}
\hline ARTICLE INFO \\
\hline Received on: 05/07/2021 \\
Accepted on: $24 / 09 / 2021$ \\
Available Online: 05/01/2022 \\
\\
\hline Key words: \\
2-arylindoles, antibacterial \\
activity, MRSA efflux \\
inhibitor, synergistic effects.
\end{tabular}

\begin{abstract}
A series of 2-arylindoles were synthesized and characterized through spectral analysis. Their antibacterial activity and synergistic effect against multidrug-resistant Staphylococcus aureus were pharmacologically studied. 2-(4-Aminophenyl)-1H-indole (4k) showed a potent inhibitory activity at $15.6 \mu \mathrm{g} / \mathrm{ml}$ against Bacillus subtilis ATCC 6633 and Salmonella typhi ATCC 19430. 2-(3-Nitrophenyl)-1H-indole (4j) at $15.6 \mu \mathrm{g} / \mathrm{ml}$ displayed a potent antibacterial activity only against $S$. typhi ATCC 19430. All the synthesized compounds elicited a synergistic effect against methicillin-resistant $S$. aureus Sp6, drug resistance, and efflux pump genes strain. 2-(2-Hydroxyphenyl)- $1 \mathrm{H}$ indoles $\mathbf{4 g}, \mathbf{4 j}$, and $\mathbf{4 k}$ were combined with tetracyclines, thereby decreasing the minimum inhibitory concentration of tetracyclines. Molecular docking showed that interaction with key amino acids occurred at the active site of the NorA efflux pump. Prediction of the toxicity using the ADMET study was also reported.
\end{abstract}

\section{INTRODUCTION}

The development of new pharmaceutical agents is one of the greatest challenges in overcoming widely spread epidermics and drug resistance. Furthermore, the ineffectiveness of medications such as antimicrobial drugs cause an increase in mortality, morbidity, and public health problems. The major components of resistance to many classes of antimicrobials are efflux mechanisms (Reygaert, 2018). In these mechanisms, efflux pump inhibitors are used to stop bacteria from pumping antibiotics out of cells, and it has been reported that 5-nitro-2-phenylindole '.....(INF55) (1) (Fig. 1) participates in the NorA efflux pump of Gram-positive bacteria and increases the susceptibility of Staphylococcus aureus to antibiotics (Markham et al., 1999). Substituents on the phenyl ring

\footnotetext{
"Corresponding Author

Waya S. Phutdhawong, Department of Chemistry, Faculty of Science, Silpakorn University, Nakhon Pathom, Thailand. E-mail: waya.sengpracha@gmail.com
}

in INF55 (1) are also important for biological activities; that is, the 2,5-dimethoxyl group on the aryl ring (2) increases the inhibitory activity of NorA (Samosorn et al., 2006). This indicated that the substituents on the phenyl ring of the 2-arylindole nucleus affect biological activity. Similarly, the substitutions of the benzene ring of the indole nucleus diverted the biological activity, which were reported in the literature (Lal and Snape, 2012; Naik et al., 2014; Williams et al., 2013). Among these, 5-methoxy-2-phenylindole (3) was reported to be a significant antibacterial activity against Gram-positive Bacillus cereus minimum inhibitory concentrations $(\mathrm{MIC})=3.9 \mu \mathrm{g} / \mathrm{ml}$. Furthermore, 5-amidine-2-arylindole bearing a 3,5-disubstituted aryl ring (4) remarkably affects the inhibition of acid-sensing ion channels (Kuduk et al., 2009). In addition, the 2-aryl-substituted indole derivative (5) is a bacterial histidine kinase inhibitor (Deschenes et al., 1999).

With the wide-ranging biological activities, the 2-arylindole scaffold is among the privileged frameworks to study the antibacterial activity and its inhibited NorA efflux pumpresistant mechanism. Thus, the goal of this study is to investigate the 2-arylindoles with various substituents on the aryl ring with 
their antibacterial activities and the ability to potentiate the activity of commercially available drugs and overcome drug resistance.

Indole is synthesized using various synthesis methods, including Fischer indole synthesis (Cho et al., 2009), Bischler synthesis (Cossío et al., 2008), Madelung cyclization (Imanishi et al., 1996), and transition metal catalyzation (Heck and Terpko, 1979; Larock and Yum, 1991). However, in terms of synthesis efficiency, these methods are limited by their requirement of several steps to be completed (Dai et al., 2001; Ezquerra et al., 1996; Rudisill and Stille, 1989; Taylor et al., 1985). Furthermore, only a few methods are available to substitute the indole ring at the $\mathrm{C} 2$ position because the $\mathrm{C} 3$ position is more reactive than the $\mathrm{C} 2$ position for electrophilic aromatic substitution. Nevertheless, an $\mathrm{N}$-protected indole can undergo organometallic substitution at the $\mathrm{C} 2$ position.

In this study, Fischer indole synthesis was applied as a simple and effective method to prepare 2-substituted indoles. The antibacterial activities of the synthesized compounds and their synergistic antibacterial potential with tetracyclines against the clinical isolate of methicillin-resistant $S$. aureus (MRSA) Sp6 were evaluated and described. Their synergistic mechanism was also investigated through molecular docking.

\section{MATERIALS AND METHODS}

\section{General information}

Melting points (m.p.) were determined using a Stuart Scientific SMP 2 m.p. apparatus and uncorrected. ${ }^{1} \mathrm{H}$ - and ${ }^{13} \mathrm{C}$-NMR spectra were obtained with (D) $\mathrm{d}_{6}$-DMSO solutions at $300 \mathrm{MHz}$ for ${ }^{1} \mathrm{H}$ and $75 \mathrm{MHz}$ for ${ }^{13} \mathrm{C}$ with a Bruker AVANCE 300 spectrometer. Tetramethylsilane was used as an internal standard. Mass spectra were recorded with a Polaris Q or Hewlett Packard 5973 mass spectrometer. Reagents and solvents were supplied by Acros, Aldrich, Fluka, and TCI.

\section{General procedure for the synthesis of 2-substituted indoles $(4 \mathbf{a}-\mathbf{m})$}

Phenylhydrazine (1) (1.5 eq.) was mixed with ketone $(2 \mathbf{a}-\mathbf{m})(1.0$ eq. $)$ in $50 \%$ acetic acid $(20 \mathrm{ml})$. The mixture was added to a beaker containing polyphosphoric acid (1.0 eq.), stirred while being heated in an oil bath for 30 minutes. The reaction mixture was cooled to room temperature and added to iced water $(50 \mathrm{ml})$ to yield a black precipitate. The precipitate was crystallized in ethanol to obtain 2-substituted indoles $(\mathbf{4 a} \mathbf{a}-\mathbf{m})$.

\section{2-Methylindole (4a)}

The title compound was synthesized from $1(0.50 \mathrm{ml}, 5.1$ $\mathrm{mmol})$ and acetone $(\mathbf{2 a})(0.5 \mathrm{ml}, 6.8 \mathrm{mmol})$ to afford $4 \mathbf{a}(0.44 \mathrm{~g}$, $66 \%$ yield) as a pale yellow substance with m.p. of $55.3^{\circ} \mathrm{C}-56.5^{\circ} \mathrm{C}$ [(Nakazaki, 1976); $\left.58^{\circ} \mathrm{C}-60^{\circ} \mathrm{C}\right] ;{ }^{1} \mathrm{H}$ NMR $\left(300 \mathrm{MHz}, \mathrm{CDCl}_{3}\right) ; \delta$ : 7.83 (br, N), 7.50 (d, $J=7.4 \mathrm{~Hz}, 1 \mathrm{H}), 7.28$ (d, $J=7.4 \mathrm{~Hz}, 1 \mathrm{H})$, $7.11(\mathrm{td}, J=1.4,7.1 \mathrm{~Hz}, 1 \mathrm{H}), 7.06(\mathrm{td}, J=1.4,7.1 \mathrm{~Hz}, 1 \mathrm{H}), 6.21$ (s, 1H), 2.45 (s, 3H) ppm.

\section{2-tert-Butyl indole (4b)}

The title compound was synthesized from $1(0.60 \mathrm{ml}$, $6.1 \mathrm{mmol})$ and 1,1,1-trimethylacetone (2b) $(0.90 \mathrm{ml}, 6.1 \mathrm{mmol})$ to afford $4 \mathbf{b}(0.49 \mathrm{~g}, 46 \%$ yield $)$ as dark solids; m.p. $71.3^{\circ} \mathrm{C}-72.8^{\circ} \mathrm{C}$
[(Fuher et al., 2019); $\left.75^{\circ} \mathrm{C}-76^{\circ} \mathrm{C}\right] ;{ }^{1} \mathrm{H}$ NMR (300 $\left.\mathrm{MHz}, \mathrm{CDCl}_{3}\right)$; $\delta: 7.94$ (br, NH), $7.54(\mathrm{~d}, J=7.6 \mathrm{~Hz}, 1 \mathrm{H}), 7.32(\mathrm{~d}, J=7.6 \mathrm{~Hz}, 1 \mathrm{H})$, $7.12(\mathrm{t}, J=7.6 \mathrm{~Hz}, 1 \mathrm{H}), 7.06(\mathrm{t}, J=7.6 \mathrm{~Hz}, 1 \mathrm{H}), 6.26(\mathrm{~s}, 1 \mathrm{H})$, $1.40(\mathrm{~s}, 9 \mathrm{H}) \mathrm{ppm}$.

\section{2-Phenyl-1H-indole (4c)}

The title compound was synthesized from $1(0.60 \mathrm{ml}$, $60 \mathrm{mmol})$ and acetophenone $(2 \mathrm{c})(0.50 \mathrm{ml}, 40 \mathrm{mmol})$ to afford $4 c(6.49 \mathrm{~g}, 84 \%$ yield $)$ as yellow crystals; m.p. $187.9^{\circ} \mathrm{C}-188.3^{\circ} \mathrm{C}$ [(Bremner et al., 2008); $\left.190.5^{\circ} \mathrm{C}-191.0^{\circ} \mathrm{C}\right] ;{ }^{1} \mathrm{H}$ NMR $(300 \mathrm{MHz}$, DMSO-d $) ; \delta: 6.90(1 \mathrm{H}, \mathrm{s}), 6.99(1 \mathrm{H}, \mathrm{t}, J=7.5 \mathrm{~Hz}), 7.10(1 \mathrm{H}, \mathrm{t}, J=$ $7.5 \mathrm{~Hz}), 7.3(1 \mathrm{H}, \mathrm{t}, J=7.8 \mathrm{~Hz}), 7.41(1 \mathrm{H}, \mathrm{d}, J=7.5 \mathrm{~Hz}), 7.46(2 \mathrm{H}$, $\mathrm{t}, J=7.8 \mathrm{~Hz}), 7.53(1 \mathrm{H}, \mathrm{d}, J=7.8 \mathrm{~Hz}), 7.86(2 \mathrm{H}, \mathrm{d}, J=7.8 \mathrm{~Hz})$, $11.53(1 \mathrm{H}, \mathrm{s}) ;{ }^{13} \mathrm{C}-\mathrm{NMR}$ (DMSO-d 6 ); $\delta: 100.0,110.9,120.3,120.7$, 112.4, 125.2, 127.7, 129.9, 129.3, 132.4, 136.8, 137.9; HRES-MS $\mathrm{m} / \mathrm{z}$ calcd for $[\mathrm{M}]^{+} \mathrm{C}_{14} \mathrm{H}_{11} \mathrm{~N}$ : 194.0970, found: 194.0966.

\section{2-(4-Methylphenyl)-1H-indole (4d)}

The title compound was synthesized from $1(6.0 \mathrm{ml}$, $60 \mathrm{mmol}$ ) and 4-methylacetonephenone (2d) (6.56 g, 40 mmol) to afford $4 \mathbf{d}(6.88 \mathrm{~g}, 83 \%$ yield $)$ as yellow crystals; m.p. $218.4^{\circ} \mathrm{C}-219.7^{\circ} \mathrm{C} ;{ }^{1} \mathrm{H}$ NMR $\left(300 \mathrm{MHz}, \mathrm{DMSO}-\mathrm{d}_{6}\right) ; \delta: 2.34(3 \mathrm{H}$, s), $6.83(1 \mathrm{H}, \mathrm{s}), 6.98(1 \mathrm{H}, \mathrm{t}, J=7.8 \mathrm{~Hz}), 7.08(1 \mathrm{H}, \mathrm{t}, J=7.8 \mathrm{~Hz})$, $7.28(2 \mathrm{H}, \mathrm{d}, J=8.1 \mathrm{~Hz}), 7.39(1 \mathrm{H}, \mathrm{d}, J=7.8 \mathrm{~Hz}), 7.56(1 \mathrm{H}, \mathrm{d}$, $J=7.8 \mathrm{~Hz}), 7.75(2 \mathrm{H}, \mathrm{d}, J=8.1 \mathrm{~Hz}), 11.47(1 \mathrm{H}, \mathrm{s}) ;) ;{ }^{13} \mathrm{C}-\mathrm{NMR}$ $\left(\right.$ DMSO$\left._{-}\right) ; \delta: 20.9,98.2,111.3,119.5,120.0,121.5,125.0,128.8$, 129.5, 129.6, 136.9, 137.1, 137.9 [(Shi et al., 2008); ${ }^{1} \mathrm{H}$ NMR (300 MHz, acetone- $\left.\mathrm{d}_{6}\right) ; \delta: 2.29(3 \mathrm{H}, \mathrm{s}), 6.80(1 \mathrm{H}, \mathrm{s}), 7.00-6.95(1 \mathrm{H}, \mathrm{m})$, $7.08-7.03(2 \mathrm{H}, \mathrm{m}), 7.23(1 \mathrm{H}, \mathrm{d}, J=7.8), 7.38-7.34(1 \mathrm{H}, \mathrm{m}), 7.52$ $(1 \mathrm{H}, \mathrm{d}, J=9.0 \mathrm{~Hz}), 7.71(2 \mathrm{H}, \mathrm{t}, J=8.4 \mathrm{~Hz}), 10.56(1 \mathrm{H}, \mathrm{s}) \cdot{ }^{13} \mathrm{C}$ NMR $(75 \mathrm{MHz}$, acetone-d $) ; \delta$ : (21.0, 99.2, 111.8, 120.2, 120.8, 122.3, 125.7, 130.2, 130.8, 137.1, 138.1)]; HRES-MS m/z calcd for $[\mathrm{M}]^{+} \mathrm{C}_{15} \mathrm{H}_{14} \mathrm{~N}$ : 208.1126, found: 208.1131.

\section{2-(3-Methoxyphenyl)-1H-indole (4e)}

The title compound was synthesized from $1(6.0 \mathrm{ml}$, $60 \mathrm{mmol}$ ) and 3-methoxyacetophenone (2e) (6.56 g, $40 \mathrm{mmol})$ to afford $4 \mathrm{e}(7.41 \mathrm{~g}, 83 \%$ yield) as dark yellow crystals; m.p. $128.9^{\circ} \mathrm{C}-130.0^{\circ} \mathrm{C} ;{ }^{1} \mathrm{H}$ NMR $\left(300 \mathrm{MHz}, \mathrm{DMSO}-\mathrm{d}_{6}\right) ; \delta: 3.84(3 \mathrm{H}$, s), $6.88(1 \mathrm{H}, \mathrm{d}, J=8.1 \mathrm{~Hz}), 6.92(1 \mathrm{H}, \mathrm{s}), 7.00(1 \mathrm{H}, \mathrm{t}, J=7.8 \mathrm{~Hz})$, $7.10(1 \mathrm{H}, \mathrm{t}, J=8.1 \mathrm{~Hz}), 7.36(1 \mathrm{H}, \mathrm{t}, J=8.1 \mathrm{~Hz}), 7.40(1 \mathrm{H}, \mathrm{d}, J$ $=8.1 \mathrm{~Hz}), 7.44(1 \mathrm{H}, \mathrm{s}), 7.45(1 \mathrm{H}, \mathrm{d}, J=7.8 \mathrm{~Hz}), 7.53(1 \mathrm{H}, \mathrm{d}$, $J=7.8 \mathrm{~Hz}), 11.53(1 \mathrm{H}, \mathrm{s}) ;{ }^{13} \mathrm{C}-\mathrm{NMR}\left(\mathrm{DMSO}_{6}\right)$; $\delta: 55.6,99.3$, $110.5,111.6,113.4,117.8,119.8,120.4,122.0,128.5,130.4$, 133.8, 137.3, 137.7, 160.0 [(Shi et al., 2008); ${ }^{1} \mathrm{H}$ NMR $(300 \mathrm{MHz}$, $\left.\mathrm{CDCl}_{3}\right) ; \delta: 3.85(3 \mathrm{H}, \mathrm{s}), 6.74(1 \mathrm{H}, \mathrm{s}), 6.84(1 \mathrm{H}, \mathrm{d}, J=8.4 \mathrm{~Hz}), 7.08$ $(1 \mathrm{H}, \mathrm{s}), 7.43-7.24(4 \mathrm{H}, \mathrm{m}), 7.61(2 \mathrm{H}, \mathrm{d}, J=7.2 \mathrm{~Hz}), 8.23(1 \mathrm{H}, \mathrm{s})$. ${ }^{13} \mathrm{C}$ NMR $\left(75 \mathrm{MHz}, \mathrm{CDCl}_{3}\right) ; \delta: 55.8,99.8,102.2,111.6,112.6$, 125.0, 127.5, 128.9, 129.7, 138.6, 152.0, 154.4]; HRES-MS m/z calcd for $[\mathrm{M}]^{+} \mathrm{C}_{15} \mathrm{H}_{14} \mathrm{NO}$ : 224.1075, found: 224.1060.

\section{2-(4-Methoxyphenyl)-1H-indole (4f)}

The title compound was synthesized from $1(6.0 \mathrm{ml}$, $60 \mathrm{mmol}$ ) and 4-methoxyacetophenone (2f) (6.56 g, $40 \mathrm{mmol})$ to afford $4 \mathbf{f}(7.14 \mathrm{~g}, 80 \%$ yield) as dark yellow crystals; m.p. $221.0^{\circ} \mathrm{C}-222.5^{\circ} \mathrm{C} ;{ }^{1} \mathrm{H}$ NMR $\left(300 \mathrm{MHz}, \mathrm{DMSO}-\mathrm{d}_{6}\right) ; \delta: 3.83(3 \mathrm{H}, \mathrm{s})$, $6.75(1 \mathrm{H}, \mathrm{s}), 6.97(1 \mathrm{H}, \mathrm{t}, J=6.9 \mathrm{~Hz}), 7.03(2 \mathrm{H}, \mathrm{d}, J=6.9 \mathrm{~Hz})$, $7.06(1 \mathrm{H}, \mathrm{t}, J=9.0 \mathrm{~Hz}), 7.37(1 \mathrm{H}, \mathrm{d}, J=6.9 \mathrm{~Hz}), 7.49(1 \mathrm{H}, \mathrm{d}$, 
$J=6.9 \mathrm{~Hz}), 7.79(2 \mathrm{H}, \mathrm{d}, J=9.0 \mathrm{~Hz}), 11.42(1 \mathrm{H}, \mathrm{s}) ;) ;{ }^{13} \mathrm{C}-\mathrm{NMR}$ $\left(\right.$ DMSO$\left._{-} \mathrm{d}_{6}\right) ; \delta: 55.4,97.5,111.2,114.5,119.4,119.8,121.2,125.0$, 126.5,1 29.0, 137.1, 138.0, 159.0 [(Shi et al., 2008); ${ }^{1} \mathrm{H}$ NMR (300 MHz, acetone- $\left.\mathrm{d}_{6}\right) ; \delta: 3.86(3 \mathrm{H}, \mathrm{s}), 6.76(1 \mathrm{H}, \mathrm{s}), 7.01-6.97(4 \mathrm{H}, \mathrm{m})$, $7.38(1 \mathrm{H}, \mathrm{d}, J=9.0), 7.53(1 \mathrm{H}, \mathrm{d}, J=7.5 \mathrm{~Hz}), 7.85-7.77(2 \mathrm{H}, \mathrm{m})$, $10.55(1 \mathrm{H}, \mathrm{s}) .{ }^{13} \mathrm{C}$ NMR $(75 \mathrm{MHz}$, acetone-d $) ; \delta: 60.4,103.5$, $116.6,120.0,125.1,125.5,126.9,131.1,132.1,135.2,143.1$, 143.8, 165.1]; HRES-MS m/z calcd for $[\mathrm{M}]^{+} \mathrm{C}_{15} \mathrm{H}_{14} \mathrm{NO}: 224.1075$, found: 224.1088 .

\section{2-(2-Hydroxyphenyl)-1H-indole (4g)}

The title compound was synthesized from $1(6.0 \mathrm{ml}$, $60 \mathrm{mmol})$ and 2-hydroxyacetophenone $(\mathbf{2 g})(4.80 \mathrm{ml}, 40 \mathrm{mmol})$ to afford $4 \mathrm{~g}$ (5.01 g, 60\% yield) as dark green crystals; m.p. $221.0^{\circ} \mathrm{C}-223.0^{\circ} \mathrm{C} ;{ }^{1} \mathrm{H}$ NMR $\left(300 \mathrm{MHz}, \mathrm{DMSO}_{-}\right.$); $\delta$ : 6.82-6.91 $(3 \mathrm{H}, \mathrm{m}), 7.04(1 \mathrm{H}, \mathrm{d}, J=8.7 \mathrm{~Hz}), 7.06(1 \mathrm{H}, \mathrm{d}, J=8.7 \mathrm{~Hz}), 7.21$ $(1 \mathrm{H}, \mathrm{t}, J=8.0 \mathrm{~Hz}), 7.28(1 \mathrm{H}, \mathrm{t}, J=8.7 \mathrm{~Hz}), 7.29(1 \mathrm{H}, \mathrm{t}, J=$ $8.7 \mathrm{~Hz}), 7.54(1 \mathrm{H}, \mathrm{d}, J=8.0 \mathrm{~Hz}), 9.43(1 \mathrm{H}, \mathrm{s}), 12.80(1 \mathrm{H}, \mathrm{s})$; ${ }^{13} \mathrm{C}-\mathrm{NMR}$ (DMSO-d $) ; \delta$ : 112.6, 116.7, 117.7, 119.0, 119.4, 120.4, $120.6,127.5,129.5,131.6,136.5,145.0,147.9,157.4[($ Snape et al., 2013); ${ }^{1} \mathrm{H}$ NMR (300 MHz; CDCl3); $\delta: 6.0-5.0(1 \mathrm{H}$, br s), $6.87(1 \mathrm{H}, \mathrm{m}, \mathrm{Ar}), 6.91(1 \mathrm{H}, \mathrm{dd}, J=0.9$ and $8.1 \mathrm{~Hz}, \mathrm{Ar}), 7.04(1 \mathrm{H}$, $\mathrm{td}, J=1.1$ and $7.6 \mathrm{~Hz}, \mathrm{Ar}), 7.26-7.11(3 \mathrm{H}, \mathrm{m}, \mathrm{Ar}), 7.42(1 \mathrm{H}, \mathrm{d}, J$ $=8.1 \mathrm{~Hz}, \mathrm{Ar}), 7.66(1 \mathrm{H}, \mathrm{d}, J=7.8 \mathrm{~Hz}, \mathrm{Ar}), 7.70(1 \mathrm{H}, \mathrm{dd}, J=1.6$ and $7.8 \mathrm{~Hz}, \mathrm{Ar}), 9.22\left(1 \mathrm{H}\right.$, br s); ${ }^{13} \mathrm{C}-\mathrm{NMR}(75 \mathrm{MHz}$; CDCl3); $\delta: 100.2,116.6,119.1,120.1,120.4,121.5,122.2,128.4,128.9$, 134.8, 136.4, 152.0]; HRES-MS m/z calcd for $[\mathrm{M}]^{+} \mathrm{C}_{14} \mathrm{H}_{12} \mathrm{NO}$ : 210.0919 , found: 210.0908 .

\section{2-(3-Hydroxyphenyl)-1H-indole (4h)}

The title compound was synthesized from 1 (6.0 $\mathrm{ml}, 60 \mathrm{mmol}$ ) and 3-hydroxyacetophenone (2h) (5.44 g, 40 $\mathrm{mmol})$ to afford $4 \mathrm{~h}$ (5.02 g, 60\% yield) as violet crystals; m.p. $220.0^{\circ} \mathrm{C}-222.0^{\circ} \mathrm{C} ;{ }^{1} \mathrm{H}$ NMR $(300 \mathrm{MHz}$, DMSO-d $)$; $\delta: 6.84(1 \mathrm{H}$, s), $6.97(1 \mathrm{H}, \mathrm{t}, J=7.8 \mathrm{~Hz}), 7.07(1 \mathrm{H}, \mathrm{t}, J=7.8 \mathrm{~Hz}), 7.13(1 \mathrm{H}, \mathrm{d}, J=$ $7.8 \mathrm{~Hz}), 7.35(1 \mathrm{H}, \mathrm{t}, J=7.8 \mathrm{~Hz}), 7.38(1 \mathrm{H}, \mathrm{t}, J=7.8 \mathrm{~Hz}), 7.51(1 \mathrm{H}$, d, $J=7.8 \mathrm{~Hz}), 7.55(1 \mathrm{H}, \mathrm{d}, J=7.8 \mathrm{~Hz}), 7.67(1 \mathrm{H}, \mathrm{s}), 11.67(1 \mathrm{H}, \mathrm{s})$; ${ }^{13} \mathrm{C}-\mathrm{NMR}$ (DMSO-d $) ; \delta$ : 99.0, 111.6, 120.1, 114.7, 116.8, 119.6, 120.2, 121.7, 128.7, 129.8, 133.5, 137.3, 137.6, 157.9 [(Kim et al., 2016); ${ }^{1} \mathrm{H}$ NMR (400 MHz, DMSO-d $) ; \delta: 6.73(1 \mathrm{H}, \mathrm{d}, J=7.6 \mathrm{~Hz})$, $6.79(1 \mathrm{H}, \mathrm{s}), 7.38(1 \mathrm{H}, \mathrm{dd}, J=8.0,2.4 \mathrm{~Hz}), 6.98(1 \mathrm{H}, \mathrm{td}, J=7.6$, $2.6 \mathrm{~Hz}), 7.08(1 \mathrm{H}, \mathrm{td}, J=7.5 \mathrm{~Hz}, 2.3 \mathrm{~Hz}), 7.31-7.20(3 \mathrm{H}, \mathrm{m}), 7.51$ $(1 \mathrm{H}, \mathrm{dd}, J=7.5,2.0 \mathrm{~Hz}), 9.55(1 \mathrm{H}, \mathrm{s}), 11.44(1 \mathrm{H}, \mathrm{s}) ;{ }^{13} \mathrm{C} \mathrm{NMR}$ (100 MHz, DMSO-d $) ; \delta: 98.5,111.3,111.9,114.5,116.0,119.3$, 120.0, 121.4, 128.6, 129.9, 133.5, 137.0, 127.8, 157.8]; HRES-MS $\mathrm{m} / \mathrm{z}$ calcd for $[\mathrm{M}]^{+} \mathrm{C}_{14} \mathrm{H}_{12} \mathrm{NO}: 210.0919$, found: 210.0934 .

\section{2-(4-Hydroxyphenyl)-1H-indole (4i)}

The title compound was synthesized from $1(6.0 \mathrm{ml}, 60$ $\mathrm{mmol})$ and 4-hydroxyacetophenone (2i) $(5.44 \mathrm{~g}, 40 \mathrm{mmol})$ to afford $4 \mathbf{i}(5.10 \mathrm{~g}, 61 \%$ yield $)$ as yellow crystals; m.p. $240.0^{\circ} \mathrm{C}-241.5^{\circ} \mathrm{C}$; ${ }^{1} \mathrm{H}$ NMR (300 MHz, DMSO-d $)$; $\delta$ : 6.68. $(1 \mathrm{H}, \mathrm{s}), 6.86(1 \mathrm{H}, \mathrm{d}, J=8.7$ $\mathrm{Hz}), 6.96(1 \mathrm{H}, \mathrm{t}, J=7.2 \mathrm{~Hz}), 7.04(1 \mathrm{H}, \mathrm{t}, J=7.2 \mathrm{~Hz}), 7.21(1 \mathrm{H}, \mathrm{s})$, $7.37(1 \mathrm{H}, \mathrm{d}, J=7.2 \mathrm{~Hz}), 7.47(1 \mathrm{H}, \mathrm{d}, J=7.2 \mathrm{~Hz}), 7.68(1 \mathrm{H}, \mathrm{d}, J$ $=8.7 \mathrm{~Hz}), 11.31(1 \mathrm{H}, \mathrm{s})\left[\left(\right.\right.$ Tsuchimoto et al., 2005); ${ }^{1} \mathrm{H}$ NMR $(300$ $\mathrm{MHz}$, acetone-d $\left.{ }_{6}\right) ; \delta: \mathrm{d} 6.71(1 \mathrm{H}, \mathrm{dd}, J=2.1,0.9 \mathrm{~Hz}),, 6.91(2 \mathrm{H}$, $\mathrm{dt}, J=9.0,2.4 \mathrm{~Hz}), 6.97(1 \mathrm{H}, \mathrm{ddd}, J=7.8,6.8,1.2 \mathrm{~Hz}), 6.97(1 \mathrm{H}$ ddd, $J=8.0,7.0,1.2 \mathrm{~Hz}), 7.33-7.38(1 \mathrm{H}, \mathrm{m}), 7.48-7.53(1 \mathrm{H}, \mathrm{m})$,
$7.69(2 \mathrm{H}, \mathrm{dt}, J=8.7,2.5 \mathrm{~Hz}), 8.52(1 \mathrm{H}, \mathrm{s}), 10.47(1 \mathrm{H}, \mathrm{bs})]$; ${ }^{13}$ C-NMR (DMSO-d $\left.{ }_{6}\right) ; \delta: 97.0,114.2,115.3,119.7,121.0,123.6$, 126.6, 126.7, 128.8, 136.0, 137.0, 157.2; HRES-MS m/z calcd for $[\mathrm{M}]^{+} \mathrm{C}_{14} \mathrm{H}_{12} \mathrm{NO}: 210.0919$, found: 210.0903 .

\section{2-(3-Nitrophenyl)-1H-indole (4j)}

The title compound was synthesized from $1(6.0 \mathrm{ml}$, $60 \mathrm{mmol}$ ) and 3-nitroacetophenone (2j) (6.56 g, $40 \mathrm{mmol})$ to afford $\mathbf{4 j}$ (7.05 g, 74\% yield) as dark yellow crystals; m.p. $169.2^{\circ} \mathrm{C}-171.3^{\circ} \mathrm{C} ;{ }^{1} \mathrm{H}$ NMR $\left(300 \mathrm{MHz}, \mathrm{DMSO}_{-} \mathrm{d}_{6}\right) ; \delta: 7.06(1 \mathrm{H}, \mathrm{t}$, $J=7.8 \mathrm{~Hz}), 7.13(1 \mathrm{H}, \mathrm{s}), 7.16(1 \mathrm{H}, \mathrm{t}, J=7.8 \mathrm{~Hz}), 7.40(1 \mathrm{H}, \mathrm{d}, J$ $=8.1 \mathrm{~Hz}), 7.57(1 \mathrm{H}, \mathrm{d}, J=7.8 \mathrm{~Hz}), 7.73(1 \mathrm{H}, \mathrm{d}, J=7.8 \mathrm{~Hz}), 8.13$ $(1 \mathrm{H}, \mathrm{d}, J=8.1 \mathrm{~Hz}), 8.83(1 \mathrm{H}, \mathrm{d}, J=8.1 \mathrm{~Hz}), 8.70(1 \mathrm{H}, \mathrm{s}), 11.42$ $(1 \mathrm{H}, \mathrm{s}) ;{ }^{13} \mathrm{C}-\mathrm{NMR}$ (DMSO-d $) ; \delta: 87.1,111.7,117.1,118.5,120.8$, 123.3, 125.1, 130.2, 132.8, 138.7, 141.7, 146.5, 148.5, 161.0 [(Shi et al., 2008); ${ }^{1} \mathrm{H}$ NMR (300 MHz, acetone- $\left.\mathrm{d}_{6}\right) ; \delta: 6.65(1 \mathrm{H}, \mathrm{s})$, 7.38-7.16 (4H, m), 7.66-7.58 (2H, m), $7.81(1 \mathrm{H}, \mathrm{d}, J=9.0), 8.21$ $(1 \mathrm{H}, \mathrm{d}, J=9.0), 8.35(1 \mathrm{H}, \mathrm{s}), 10.53(1 \mathrm{H}, \mathrm{s}) ;{ }^{13} \mathrm{C}-\mathrm{NMR}(75 \mathrm{MHz}$, acetone- $\left.\mathrm{d}_{6}\right) ; \delta: 103.2,109.8,120.3,120.8,122.6,123.6,127.6$, $129.5,134.4,134.9,138.7,148.3]$; HRES-MS m/z calcd for $[\mathrm{M}]^{+}$ $\mathrm{C}_{14} \mathrm{H}_{11} \mathrm{~N}_{2} \mathrm{O}_{2}: 239.0821$, found: 239.0843 .

\section{2-(4-Nitrophenyl)-1H-indole (4k)}

The title compound was synthesized from $1(6.0 \mathrm{ml}$, $60 \mathrm{mmol}$ ) and 4-nitroacetophenone (2k) (6.56 g, $40 \mathrm{mmol})$ to afford $4 \mathbf{k}$ (7.60 g, $80 \%$ yield) as dark yellow crystals; m.p. $173.2^{\circ} \mathrm{C}-175.3^{\circ} \mathrm{C}$ [(Westwell et al., 2013); $\left.168^{\circ} \mathrm{C}-172^{\circ} \mathrm{C}\right] ;{ }^{1} \mathrm{H}$ NMR (300 MHz, DMSO-d $)$; $\delta: 6.87(1 \mathrm{H}, \mathrm{s}), 6.99(1 \mathrm{H}, \mathrm{t}, J=7.5$ $\mathrm{Hz}), 7.09(1 \mathrm{H}, \mathrm{t}, J=7.5 \mathrm{~Hz}), 7.31(2 \mathrm{H}, \mathrm{d}, J=9.0 \mathrm{~Hz}), 7.38(1 \mathrm{H}$, $\mathrm{d}, J=7.5 \mathrm{~Hz}), 7.53(1 \mathrm{H}, \mathrm{d}, J=7.5 \mathrm{~Hz}), 7.90(2 \mathrm{H}, \mathrm{d}, J=9.0 \mathrm{~Hz})$, $11.42(1 \mathrm{H}, \mathrm{s}) ;{ }^{13} \mathrm{C}-\mathrm{NMR}$ (DMSO-d 6 ); $\delta: 102.5,111.7,119.9,120.8$, $123.1,124.36,125.5,128.4,135.2,137.9,138.6,145.8$; HRESMS m/z calcd for $[\mathrm{M}]^{+} \mathrm{C}_{14} \mathrm{H}_{11} \mathrm{~N}_{2} \mathrm{O}_{2}: 239.0821$, found: 239.0803 .

\section{2-(4-Fluorophenyl)-1H-indole (4l)}

The title compound was synthesized from $1(6.0 \mathrm{ml}$, $60 \mathrm{mmol})$ and 4-fluoroacetophenone (2l) $(0.50 \mathrm{ml}, 40 \mathrm{mmol})$ to afford $4 \mathrm{l}(6.80 \mathrm{~g}, 80 \%$ yield) as dark green crystals; m.p. $172.9^{\circ} \mathrm{C}-173.5^{\circ} \mathrm{C} ;{ }^{1} \mathrm{H}$ NMR $\left(300 \mathrm{MHz}, \mathrm{DMSO}-\mathrm{d}_{6}\right) ; \delta: 6.87(1 \mathrm{H}, \mathrm{s})$, $6.99(1 \mathrm{H}, \mathrm{t}, J=7.5 \mathrm{~Hz}), 7.09(1 \mathrm{H}, \mathrm{t}, J=7.5 \mathrm{~Hz}), 7.31(2 \mathrm{H}, \mathrm{dd}, J=$ 9.0, $9.0 \mathrm{~Hz}), 7.38(1 \mathrm{H}, \mathrm{d}, J=7.5 \mathrm{~Hz}), 7.53(1 \mathrm{H}, \mathrm{d}, J=7.5 \mathrm{~Hz}), 7.90$ $(2 \mathrm{H}, \mathrm{dd}, J=9.0,5.4 \mathrm{~Hz}), 11.42(1 \mathrm{H}, \mathrm{s}) ;{ }^{13} \mathrm{C}-\mathrm{NMR}\left(\mathrm{DMSO}_{\mathrm{d}}\right) ; \delta$ : 99.2, 111.8, 116.2, 116.5,120.0, 120.6, 122.2, 127.4, 127.5, 129.1, 137.2, 137.69 [(Shi et al., 2008); ${ }^{1} \mathrm{H}$ NMR (300 MHz, acetone-d 6 ); $\delta: 6.84(1 \mathrm{H}, \mathrm{s}), 7.24-7.01(3 \mathrm{H}, \mathrm{m}), 7.42(1 \mathrm{H}, \mathrm{d}, J=10.2 \mathrm{~Hz}), 7.56$ $(1 \mathrm{H}, \mathrm{d}, J=8.1 \mathrm{~Hz}), 7.89-7.84(1 \mathrm{H}, \mathrm{m}), 10.58(1 \mathrm{H}, \mathrm{s}) ;{ }^{13} \mathrm{C} \mathrm{NMR}$ (75 MHz, acetone-d $)$; $\delta$ : 99.6, 111.7, 116.1, 116.3, 120.2, 122.4, 127.5, 127.6, 129.9, 138.1, 164.3]; HRES-MS m/z calcd for $[\mathrm{M}]^{+}$ $\mathrm{C}_{14} \mathrm{H}_{11} \mathrm{FN}: 212.0876$, found: 212.0853 .

\section{2-(4-Aminophenyl)-1H-indole (4m)}

The title compound was synthesized from $1(6.0 \mathrm{ml}$, $60 \mathrm{mmol})$ and 4-aminoacetophenone (2m) (5.40 g, $40 \mathrm{mmol})$ to afford $4 \mathrm{~m}$ (6.99 g, 84\% yield) as dark green crystals; m.p. $261.4^{\circ} \mathrm{C}-262.8^{\circ} \mathrm{C}\left[(\right.$ Westwell et al., 2013$\left.) ; 168^{\circ} \mathrm{C}-172^{\circ} \mathrm{C}\right] ;{ }^{1} \mathrm{H}$ NMR (300 MHz, DMSO-d $)$; $\delta: 6.71(1 \mathrm{H}, \mathrm{s}), 6.67-7.00(2 \mathrm{H}, \mathrm{br})$, $6.96(1 \mathrm{H}, \mathrm{t}, J=7.8 \mathrm{~Hz}), 6.97(2 \mathrm{H}, \mathrm{d}, J=7.8 \mathrm{~Hz}), 7.04(1 \mathrm{H}, \mathrm{t}, J=$ $7.8 \mathrm{~Hz}), 7.36(1 \mathrm{H}, \mathrm{d}, J=7.8 \mathrm{~Hz}), 7.46(1 \mathrm{H}, \mathrm{d}, J=7.8 \mathrm{~Hz}), 7.77$ 
$(2 \mathrm{H}, \mathrm{d}, J=7.8 \mathrm{~Hz}), 11.39(1 \mathrm{H}, \mathrm{s})\left[\left(\right.\right.$ Westwell et al., 2013); ${ }^{1} \mathrm{H}$ NMR $\left(500 \mathrm{MHz}, \mathrm{DMSO}-d_{6}\right) ; \delta: 5.27(2 \mathrm{H}, \mathrm{s}), 6.57(1 \mathrm{H}, \mathrm{s}), 6.64(2 \mathrm{H}, \mathrm{d}$, $J=8.4 \mathrm{~Hz}), 6.93(1 \mathrm{H}, \mathrm{d}, J=8.5 \mathrm{~Hz}), 7.00(1 \mathrm{H}, \mathrm{m}), 7.32(1 \mathrm{H}, \mathrm{d}$, $J=8.2 \mathrm{~Hz}), 7.43(1 \mathrm{H}, \mathrm{d}, J=8.8 \mathrm{~Hz}), 7.52(2 \mathrm{H}, \mathrm{d}, J=8.4 \mathrm{~Hz})$, $11.16(1 \mathrm{H}, \mathrm{s})] ;{ }^{13} \mathrm{C}-\mathrm{NMR}\left(\mathrm{DMSO}-\mathrm{d}_{6}\right) ; \delta: 95.9,111.1,114.6,119.3$, $119.5,120.6,120.7,126.3,129.3,136.9,139.3,148.0 ;$ HRES-MS $\mathrm{m} / \mathrm{z}$ calcd for $[\mathrm{M}]^{+} \mathrm{C}_{14} \mathrm{H}_{13} \mathrm{~N}_{2}: 209.1079$, found: 209.1090

\section{General procedure for the amidation of $4 \mathrm{~m}$ with acid bromide derivatives $(6 a-f)$}

Acid bromide (5) (1.7 eq.) was added to a solution of 2-(4-aminophenyl)-1 $H$-indole (4m) (1.0 eq.) and $\mathrm{Et}_{3} \mathrm{~N}$ (0.8 eq.) in dry THF $(2.5 \mathrm{ml})$ at $0^{\circ} \mathrm{C}$. The mixture was stirred for 2 hours and water $(25 \mathrm{ml})$ was added. The mixture was extracted with $\mathrm{CH}_{2} \mathrm{Cl}_{2}$ $(3 \times 20 \mathrm{ml})$. The organic phase was washed with sat. $\mathrm{NaHCO}_{3}$ and dried with anh. $\mathrm{Na}_{2} \mathrm{SO}_{4}$. Then, the solvent was evaporated under vacuum and purified via column chromatography (silica gel, 1:2 hexane:EtOAc) to give the amide derives $(\mathbf{6 a}-\mathbf{f})$.

\section{2-(4-Acetamidophenyl)-1H-indole (6a)}

The title compound was synthesized from $4 \mathrm{~m}$ (75 mg, $0.36 \mathrm{mmol}), \mathrm{Et}_{3} \mathrm{~N}(0.05 \mathrm{ml}, 0.36 \mathrm{mmol})$, and acetyl bromide $(0.05$ $\mathrm{ml}, 0.6 \mathrm{mmol})$ to obtain 2-(4-acetamidophenyl)- $1 H$-indole (6a) (47.8 mg, 53\% yield) as a brown solid; m.p. $286.5^{\circ} \mathrm{C}-288.2^{\circ} \mathrm{C}$ [(Ackermann et al., 2009); $283.9^{\circ} \mathrm{C}-286.8^{\circ} \mathrm{C}$ ]; ${ }^{1} \mathrm{H}$ NMR $(300$ MHz, DMSO-d $)$; $\delta$ : 11.42 (br, NH), 10.04 (br, NH), 7.75 (d, $J$ $=8.7 \mathrm{~Hz}, 2 \mathrm{H}), 7.65(\mathrm{~d}, J=8.7 \mathrm{~Hz}, 2 \mathrm{H}), 7.49(\mathrm{~d}, J=7.7 \mathrm{~Hz}, 1 \mathrm{H})$, $7.37(\mathrm{~d}, J=8.0 \mathrm{~Hz}, 1 \mathrm{H}), 7.07$ (t, $J=8.0 \mathrm{~Hz}, 1 \mathrm{H}), 6.97$ (t, $J=7.9$ $\mathrm{Hz}, 1 \mathrm{H}), 6.79$ (s, 1H), 2.07 (s, 3H) ppm; ${ }^{13} \mathrm{C}-\mathrm{NMR}$ (DMSO-d ${ }_{6}$ ); $\delta: 21.4\left(\mathrm{CH}_{3}\right), 96.5,111.9,114.5(\times 2), 118.6,119.3,120.7,121.7$, $127.7(\times 2), 128.9,136.0,139.5,147.8,172.5$ [(Ackermann et al., 2009); ${ }^{1} \mathrm{H}-\mathrm{NMR}\left(300 \mathrm{MHz}, \mathrm{DMSO}-\mathrm{d}_{6}\right) ; \delta: 2.10(3 \mathrm{H}, \mathrm{s}), 6.80(1 \mathrm{H}$, $\mathrm{m}), 7.02-6.96(1 \mathrm{H}, \mathrm{m}), 7.11-7.06(1 \mathrm{H}, \mathrm{m}), 7.42-7.40(1 \mathrm{H}, \mathrm{m})$, $7.52(1 \mathrm{H}, \mathrm{d}, J=7.7 \mathrm{~Hz}), 7.71(2 \mathrm{H}, \mathrm{d}, J=8.6 \mathrm{~Hz}), 7.81(2 \mathrm{H}, \mathrm{d}$, $J=8.6 \mathrm{~Hz}), 10.04(1 \mathrm{H}, \mathrm{s}), 11.42(1 \mathrm{H}, \mathrm{s}) .{ }^{13} \mathrm{C}-\mathrm{NMR}(126 \mathrm{MHz}$, DMSO-d $)_{6} ; \delta: 24.0,97.8,111.1,119.2,119.2,119.7,121.2,125.4$, 127.0, 128.8, 137.0, 137.7, 138.6, 168.3]; HRES-MS m/z calcd for $[\mathrm{M}]^{+} \mathrm{C}_{16} \mathrm{H}_{14} \mathrm{~N}_{2} \mathrm{O}: 250.1106$, found 250.1079.

\section{2-(4-Propioamidophenyl)-1H-indole (6b)}

The title compound was synthesized from 4 m $(64 \mathrm{mg}$, $0.31 \mathrm{mmol}), \mathrm{Et}_{3} \mathrm{~N}(0.05 \mathrm{ml}, 0.4 \mathrm{mmol})$, and propanoyl chloride $(0.05 \mathrm{ml}, 0.6 \mathrm{mmol})$ to obtain 2-(4-propioamidophenyl)-1Hindole (6b) (50.0 mg, 62\% yield) as a pale orange solid; m.p. $256.1^{\circ} \mathrm{C}-257.2^{\circ} \mathrm{C} ;{ }^{1} \mathrm{H}$ NMR (300 MHz, DMSO-d $) ; \delta: 1.11(3 \mathrm{H}, \mathrm{t}$, $J=7.5 \mathrm{~Hz}), 2.35(2 \mathrm{H}, \mathrm{q}, J=7.5 \mathrm{~Hz}), 6.81(1 \mathrm{H}, \mathrm{s}), 6.98(1 \mathrm{H}, \mathrm{t}, J=$ $7.4 \mathrm{~Hz}), 7.07(1 \mathrm{H}, \mathrm{t}, J=8.0 \mathrm{~Hz}), 7.38(1 \mathrm{H}, \mathrm{d}, J=8.0 \mathrm{~Hz}), 7.50(1 \mathrm{H}$, $\mathrm{d}, J=8.0 \mathrm{~Hz}), 7.68(2 \mathrm{H}, \mathrm{d}, J=8.4 \mathrm{~Hz}), 7.79(2 \mathrm{H}, \mathrm{d}, J=8.4 \mathrm{~Hz})$, 9.97 (1H, br, NH), $11.45\left(1 \mathrm{H}\right.$, br, NH); ${ }^{13} \mathrm{C}-\mathrm{NMR}$ (DMSO-d $)$ ); $\delta$ : 9.5, 26.6, 96.8, 111.1, $114.8(\times 2), 119.4,119.8,120.7,121.5,127.6$ $(\times 2), 129.4,135.6,138.5,148.2,173.2 ; \mathrm{HRES}-\mathrm{MS} \mathrm{m} / \mathrm{z}$ calcd for $[\mathrm{M}]^{+} \mathrm{C}_{17} \mathrm{H}_{16} \mathrm{~N}_{2} \mathrm{O}: 264.1263$, found 264.1222.

\section{2-(4-Octanamidophenyl)-1H-indole (6c)}

The title compound was synthesized from $4 \mathrm{~m}(70 \mathrm{mg}$, $0.34 \mathrm{mmol}), \mathrm{Et}_{3} \mathrm{~N}(0.05 \mathrm{ml}, 0.36 \mathrm{mmol})$, and octanoyl chloride $(0.10 \mathrm{ml}, 0.59 \mathrm{mmol})$ to obtain 2-(4-octanamidophenyl)-1H-indole (6c) (50 mg, 44\% yield) as a white solid; m.p. $239.2^{\circ} \mathrm{C}-240.2^{\circ} \mathrm{C}$;
${ }^{1} \mathrm{H}$ NMR (300 MHz, DMSO-d $) ; \delta: 0.88(3 \mathrm{H}, \mathrm{t}, J=6.9 \mathrm{~Hz}), 1.35-$ $1.20(8 \mathrm{H}, \mathrm{m}), 1.70-1.55(2 \mathrm{H}, \mathrm{m}), 2.33(2 \mathrm{H}, \mathrm{t}, J=7.4 \mathrm{~Hz}, 2 \mathrm{H}), 6.80$ $(1 \mathrm{H}, \mathrm{s}), 6.98(1 \mathrm{H}, \mathrm{t}, J=7.0 \mathrm{~Hz}), 7.07(1 \mathrm{H}, \mathrm{t}, J=7.0 \mathrm{~Hz}), 7.38(1 \mathrm{H}$, $\mathrm{d}, J=8.0 \mathrm{~Hz}), 7.50(1 \mathrm{H}, \mathrm{d}, J=7.8 \mathrm{~Hz}), 7.68(2 \mathrm{H}, \mathrm{d}, J=8.7 \mathrm{~Hz})$, $7.78(2 \mathrm{H}, \mathrm{d}, J=8.7 \mathrm{~Hz}), 9.97(1 \mathrm{H}, \mathrm{br}, \mathrm{NH}), 11.43(1 \mathrm{H}, \mathrm{br}, \mathrm{NH})$; ${ }^{13} \mathrm{C}-\mathrm{NMR}\left(\mathrm{DMSO}-\mathrm{d}_{6}\right) ; \delta: 14.1,25.5,29.2,29.5(\times 2), 31.7,33.7$, $40.5,111.1,114.8(\times 2), 119.4,119.8,120.7,121.5,127.6(\times 2)$, 129.4, 135.6, 138.5, 148.2, 173.2; HRES-MS m/z calcd for $[\mathrm{M}]^{+}$ $\mathrm{C}_{21} \mathrm{H}_{24} \mathrm{~N}_{2} \mathrm{O}: 320.1889$, found 320.1875 .

\section{2-(4-Benzamidophenyl)-1H-indole (6d)}

The title compound was synthesized from $\mathbf{4 m}(92.3$ $\mathrm{mg}, 0.44 \mathrm{mmol}), \mathrm{Et}_{3} \mathrm{~N}(0.1 \mathrm{ml}, 0.72 \mathrm{mmol})$, and benzoyl chloride $(0.10 \mathrm{ml}, 0.86 \mathrm{mmol})$ to obtain 2-(4-benzamidophenyl)-1H-indole (6d) $(0.12 \mathrm{~g}, 90 \%$ yield $)$ as a brown solid; m.p. $281.7^{\circ} \mathrm{C}-282.8^{\circ} \mathrm{C}$; ${ }^{1} \mathrm{H}$ NMR $(300 \mathrm{MHz}$, DMSO-d $) ; \delta: 6.01(1 \mathrm{H}, \mathrm{s}), 6.14(1 \mathrm{H}, \mathrm{t}, J$ $=7.5 \mathrm{~Hz}), 6.24(1 \mathrm{H}, \mathrm{t}, J=7.3 \mathrm{~Hz}), 6.55(1 \mathrm{H}, \mathrm{d}, J=9.0 \mathrm{~Hz})$, 6.81-6.64 (4H, m), 7.07-6.98 (4H, m), $7.14(2 \mathrm{H}, \mathrm{d}, J=7.2 \mathrm{~Hz})$, $9.51\left(1 \mathrm{H}\right.$, br, NH), $10.63(1 \mathrm{H}, \mathrm{br}, \mathrm{NH}) ;{ }^{13} \mathrm{C}-\mathrm{NMR}$ (DMSO-d $)$; $\delta$ : $96.8,111.1,114.7(\times 2), 119.6,119.8,120.7,121.7,126.9(\times 2)$, $128.6(\times 2), 130.0,132.1,134.2,136.0,136.3,137.6,137.9,148.5$, 171.6; HRES-MS m/z calcd for $[\mathrm{M}]^{+} \mathrm{C}_{21} \mathrm{H}_{16} \mathrm{~N}_{2} \mathrm{O}$ : 312.1263 , found 312.1249 .

\section{2-(2-Nitrobenzamidophenyl)-1H-indole (6e)}

The title compound was synthesized from $4 \mathbf{m}(75.0 \mathrm{mg}$, $0.36 \mathrm{mmol}), \mathrm{Et}_{3} \mathrm{~N}(0.05 \mathrm{ml}, 0.36 \mathrm{mmol}, 0.8$ eq. $)$, and 4-nitrobenzyl chloride $(0.11 \mathrm{~g}, 0.63 \mathrm{mmol})$ to obtain 2-(2-nitrobenzamidophenyl)$1 \mathrm{H}$-indole (6e) (0.10 g, 88\% yield) as a yellow solid; ${ }^{1} \mathrm{H}$ NMR (300 MHz, DMSO-d $)$; $\delta: 6.91(1 \mathrm{H}, \mathrm{s}), 7.03(1 \mathrm{H}, \mathrm{t}, J=7.5 \mathrm{~Hz}), 7.12$ $(1 \mathrm{H}, \mathrm{t}, J=7.7 \mathrm{~Hz}), 7.43(1 \mathrm{H}, \mathrm{d}, J=9.0 \mathrm{~Hz}), 7.56(1 \mathrm{H}, \mathrm{d}, J=9.0$ $\mathrm{Hz}), 7.92(4 \mathrm{H}, \mathrm{s}), 8.26(2 \mathrm{H}, \mathrm{d}, J=9.0 \mathrm{~Hz}), 8.43(2 \mathrm{H}, \mathrm{d}, J=9.0 \mathrm{~Hz})$, $10.74\left(1 \mathrm{H}\right.$, br, NH), $11.55\left(1 \mathrm{H}\right.$, br, NH); ${ }^{13} \mathrm{C}-\mathrm{NMR}$ (DMSO-d 6 ); $\delta: 107.9,111.1,113.2,113.8,120.2,120.5,121.0,122.2,126.1$, 129.0, 136.92, 136.97, 147.2, 152.0, 171.9; HRES-MS m/z calcd for $[\mathrm{M}]^{+} \mathrm{C}_{21} \mathrm{H}_{15} \mathrm{~N}_{3} \mathrm{O}_{3}: 357.1113$, found 357.1100.

\section{2-(4-Nitrobenzamidophenyl)-1H-indole (6f)}

The title compound was synthesized from $\mathbf{4 m}(0.11$ g, $0.52 \mathrm{mmol}), \mathrm{Et}_{3} \mathrm{~N}(0.05 \mathrm{ml}, 0.36 \mathrm{mmol})$, and 4-nitrobenzyl chloride (0.05 g, $1 \mathrm{mmol})$ to obtain 2-(4-nitrobenzamidophenyl)$1 \mathrm{H}$-indole (6f) $(88.0 \mathrm{mg}, 47 \%$ yield) as a yellow solid; m.p. $263.4^{\circ} \mathrm{C}-264.4^{\circ} \mathrm{C} ;{ }^{1} \mathrm{H}$ NMR $(300 \mathrm{MHz}$, DMSO-d $)$; $\delta: 6.86(1 \mathrm{H}$, s), $7.00(1 \mathrm{H}, \mathrm{t}, J=7.4 \mathrm{~Hz}), 7.10(1 \mathrm{H}, \mathrm{t}, J=7.5 \mathrm{~Hz}), 7.40(1 \mathrm{H}$, d, $J=8.1 \mathrm{~Hz}), 7.53(1 \mathrm{H}, \mathrm{d}, J=7.8 \mathrm{~Hz}), 7.84-7.71(4 \mathrm{H}, \mathrm{m}, 4 \mathrm{H})$, $7.95-7.84(3 \mathrm{H}, \mathrm{m}), 8.18(1 \mathrm{H}, \mathrm{d}, J=7.5 \mathrm{~Hz}), 10.79(1 \mathrm{H}, \mathrm{br}, \mathrm{NH})$, $11.48(1 \mathrm{H}, \mathrm{br}, \mathrm{NH}) ;{ }^{13} \mathrm{C}-\mathrm{NMR}$ (DMSO-d $\left.{ }_{6}\right) ; \delta: 107.8,110.9,113.1$, $113.8,120.0,120.4,121.0,122.1,126.1,128.9,136.8,136.9$, 147.1, 152.0, 171.8; HRES-MS m/z calcd for $[\mathrm{M}]^{+} \mathrm{C}_{21} \mathrm{H}_{15} \mathrm{~N}_{3} \mathrm{O}_{3}$ : 357.1113 , found 357.1105 .

\section{Determination of antibacterial activity}

The MICs of $\mathbf{4 a}-\mathbf{4} \mathbf{m}$ and $\mathbf{6 a}-\mathbf{6} \mathbf{f}$ were tested against S. aureus ATCC 25932, B. cereus ATCC 7064, Bacillus subtilis ATCC 6633, Escherichia coli ATCC 10536, and Salmonella typhi ATCC 19430 and determined with National Committee for Clinical Laboratory Standards (NCCLS) microbroth dilution methods (National Committee for Clinical Laboratory 
Standards, 2000). Kanamycin and chloramphenicol were used as references for antibacterial activity. The test samples were dissolved in DMSO with a range of 512-0.98 $\mu \mathrm{g} / \mathrm{ml}$ in nutrient broth supplemented with $10 \%$ glucose and $0.05 \%$ phenol red (NBGP). Then, $100 \mu \mathrm{l}$ of each concentration was added to each well (96-well microplate) containing $95 \mu \mathrm{l}$ of NBGP and $5 \mu 1$ of inoculum (standardized at $1.5 \times 10^{6} \mathrm{cfu} / \mathrm{ml}$ by adjusting the optical density to 0.1 at $600 \mathrm{~nm}$. The final concentration of DMSO in the well was less than $1 \%$ preliminary analyses with $1 \%(\mathrm{v} / \mathrm{v})$ DMSO/NBGP affected neither the growth of the test organisms nor the change in color because of this growth). The negative control well contained $195 \mu \mathrm{l}$ of NBGP and $5 \mu \mathrm{l}$ of the standard inoculum. The plates were covered with a sterile plate sealer, agitated using a plate shaker to mix the content of the wells, and incubated at $37^{\circ} \mathrm{C}$ for 24 hours. The assay was repeated twice, and microbial growth was determined by observing the change in the color of the content of the wells (i.e., red = without growth, yellow with growth). The lowest concentration showing no color change was considered the MIC.

\section{Synergistic antibacterial assay}

MRSA Sp6 was isolated from pus samples of patients in Nakhon Pathom Hospital (Taechowisan et al., 2018). The drug resistance of this strain was detected via a cefoxitin paper method of the American Association of Clinical and Laboratory Standards Institute (CLSI, 2014). Its drug resistance and efflux pump genes were also detected through PCR amplification. The primers of the species-specific $S$. aureus (fem $A$ ) gene, the penicillin-binding protein 2 ( located tetracycline $(t e t K)$ gene were utilized for PCR assay as previously described (Taechowisan et al., 2018).

The MICs of methicillin and tetracycline were determined using a twofold dilution method in accordance with the guidelines of the National Committee for Clinical Laboratory Standards (1997). In this procedure, $100 \mathrm{ml}$ of MRSA Sp6 $\left(10^{5}\right.$ $\mathrm{cfu} / \mathrm{ml}$ ) was mixed with different concentrations of methicillin and tetracycline $(16,32,64$, and $128 \mathrm{mg} / \mathrm{ml})$ in a 96-well plate and incubated at $37^{\circ} \mathrm{C}$ for 24 hours. A control group was prepared by culturing MRSA Sp6 with $0 \mathrm{mg} / \mathrm{ml}$ methicillin and tetracycline. Various concentrations $(16,32,64$, and $128 \mathrm{mg} / \mathrm{ml})$ were applied to $100 \mathrm{ml}$ of MRSA Sp6 $\left(10^{5} \mathrm{cfu} / \mathrm{ml}\right)$ cells alone or in combination with either methicillin or tetracycline $(16,32,64$, and $128 \mathrm{mg} /$ $\mathrm{ml}$ ) in 96-well plates to elucidate the effect of methicillin and tetracycline activities in the presence of 2-arylindole derivatives. The plates were incubated at $37^{\circ} \mathrm{C}$ for 24 hours. A blank control was also prepared by culturing MRSA Sp6 cells in media only without methicillin and tetracycline or 2-arylindole derivatives.

\section{Molecular modeling}

The homology model of NorA from $S$. aureus was constructed using a Swiss-Model server and the protein EmrD efflux pump from $E$. coli (PDB ID: 2GFP) because the crystal structure of NorA from $S$. aureus remained unavailable (Zárate et al., 2019). Molecular docking was performed with iGEMDOCK v2.1 (Hsu et al., 2011) to explore the protein-substrate interactions and binding position of $\mathbf{4 g}-\mathbf{k}$ and $\mathbf{4} \mathbf{m}$ in the active site of NorA from $S$. aureus. Ciprofloxacin, which is known as a good inhibitor of NorA efflux pumps, was also docked into the NorA efflux pump, and its binding energy was compared with those of our compounds.

\section{Prediction of ADMET by computational analysis}

The computational prediction of the compounds was performed through the online software SwissADME (http:// swissadme.ch) in order to evaluate the pharmacokinetics of the synthesized molecules. The interaction of the synthesized molecules with CYP was used to predict the toxicity properties. Passive human gastrointestinal absorption (HIA) and blood-brain barrier $(\mathrm{BBB})$ were used to predict the pharmacokinetic behaviors of these molecules. Caco-2-permeability was considered as druglikeness property.

\section{RESULTS AND DISCUSSION}

\section{Chemistry}

2-Arylindoles were prepared in a manner similar to previously reported Fischer indole synthesis (Hughes, 1993)

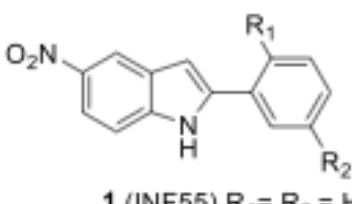

$\mathrm{R}_{2}=\mathrm{H}$

$2 \mathrm{R}_{1}=\mathrm{R}_{2}=\mathrm{OMe}$

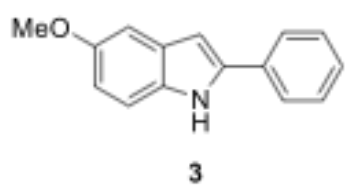

3

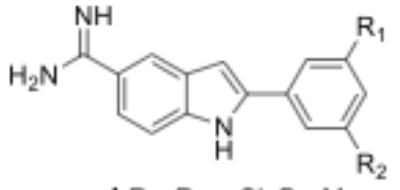

$4 \mathrm{R}_{1}, \mathrm{R}_{2}=\mathrm{Cl}, \mathrm{Br}, \mathrm{Me}$

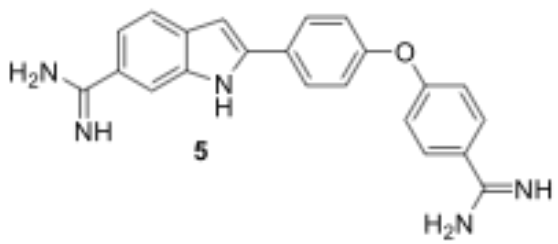

Figure 1. Bioactive 2-arylindole. 
Table 1. Synthesis of 2-substituted phenyl-1 $H$ indole derivatives.

\begin{tabular}{|c|c|c|c|c|c|}
\hline Entry & Ketone & $\mathbf{R}_{1}$ & $\begin{array}{c}\text { Time } \\
\text { (minute) }\end{array}$ & $\operatorname{Temp}\left({ }^{\circ} \mathrm{C}\right)$ & Product (\%) \\
\hline 1 & $2 a$ & $\mathrm{CH}_{3}$ & 15 & 80 & 4a (66) \\
\hline 2 & $2 b$ & $\mathrm{C}\left(\mathrm{CH}_{3}\right)_{3}$ & 15 & 80 & $4 b(46)$ \\
\hline 3 & $2 c$ & $\mathrm{Ph}$ & 15 & 80 & $4 \mathbf{c}(88)$ \\
\hline 4 & $2 d$ & $p-\mathrm{CH}_{3} \mathrm{Ph}$ & 30 & 100 & $4 d(83)$ \\
\hline 5 & $2 e$ & $m-\mathrm{OCH}_{3} \mathrm{Ph}$ & 120 & 100 & $4 e(73)$ \\
\hline 6 & $2 f$ & $p-\mathrm{OCH}_{3} \mathrm{Ph}$ & 240 & 100 & $4 f(70)$ \\
\hline 7 & $2 \mathrm{~g}$ & $o$-phenol & 240 & 100 & $4 \mathrm{~g}(60)$ \\
\hline 8 & $2 \mathrm{~h}$ & $m$-phenol & 120 & 100 & $4 h(60)$ \\
\hline 9 & $2 \mathbf{i}$ & $p$-phenol & 240 & 100 & $4 i(61)$ \\
\hline 10 & $2 j$ & $m-\mathrm{NO}_{2} \mathrm{Ph}$ & 10 & 75 & $4 \mathbf{j}(74)$ \\
\hline 11 & $2 k$ & $p-\mathrm{NO}_{2} \mathrm{Ph}$ & 5 & 70 & $4 \mathbf{k}(80)$ \\
\hline 12 & 21 & $p$-FPh & 30 & 100 & $41(80)$ \\
\hline 13 & $2 m$ & $p-\mathrm{NH}_{2} \mathrm{Ph}$ & 60 & 100 & $4 m(84)$ \\
\hline
\end{tabular}

(Scheme 1). After the reaction parameters were screened, the reaction of 1 (1.5 eq.) and methyl ketone derivatives (2;1.0 eq.) in $50 \%$ acetic acid $(20 \mathrm{ml})$ produced moderate to high yields of indole derivatives (4) via hydrazone derivatives (3). The reaction time and temperature of each product under the optimized conditions are shown in Table 1. The heating duration of most of the aromatic derivatives of $\mathbf{2}$ at high temperatures should be prolonged. Unidentified products except nitrobenzene substitution (entries 10-11) were obtained at high temperatures (Scheme 2). The structures of all known compounds were confirmed by m.p. and spectroscopic analysis.

The substitutions of amino groups on the aromatic ring were investigated to study the structure-activity relationship (Scheme 2). Amidation using acid halide (5) produced moderated yields of amides $(\mathbf{6 a}-\mathbf{f})$. Then, the antibacterial activities and synergistic effects of the synthesized 2-arylindole derivatives were further evaluated.

\section{Antibacterial activity}

All the synthesized compounds $\mathbf{4 a}-\mathbf{m}$ and $\mathbf{6 a}-\mathbf{f}$ were tested against $S$. aureus ATCC 25932, B. cereus ATCC 7064, B. subtilis ATCC 6633, E. coli ATCC 10536, and S. typhi ATCC 19430 by NCCLS microbroth dilution methods (National Committee for Clinical Laboratory Standards, 1997). Kanamycin and chloramphenicol were used as positive controls, and a solvent was used as a negative control. The results are shown in Table 2 as MIC $(\mu \mathrm{g} / \mathrm{ml})$ unit. Among the compounds tested, $4 \mathbf{m}$ was the most active with an MIC of $15.6 \mu \mathrm{g} / \mathrm{ml}$ against $B$. subtilis and $S$. typhi. Furthermore, $\mathbf{4 c}, \mathbf{4 d}$, and $\mathbf{4 f}$ selectively inhibited B. subtilis and had MICs of 31,125 , and $62 \mu \mathrm{g} / \mathrm{ml}$, respectively. In addition, $\mathbf{4} \mathbf{j}$ showed antibacterial activities against $S$. typhi at $15.6 \mu \mathrm{g} / \mathrm{ml}$. Thus, the phenyl ring, substituents, and their positions on the phenyl ring are essential for potency. Compounds $4 \mathbf{e}$ and 4h with phenyl-bearing electron-donating groups on the metaposition have weak or no activity, while compound $4 \mathbf{k}$ bearing electron-withdrawing group on the meta-position has selective activity against $S$. typhi. On the other hand, the phenyl-bearing hydrophilic substituents at para-position seem to be important for the activity. The protected amino derivatives $(\mathbf{6 a}-\mathbf{f})$ had weak or no antibacterial activity against all the tested bacteria. This may assume that the amide linkage of $\mathbf{4 m}$ affected the hydrophilicity and might obstruct the interaction of these molecules and the bacterial active site.

\section{Synergistic effect}

The synthesized compounds were assayed to examine their antibacterial activities against MRSA Sp6. The results revealed that none of them exhibited an inhibitory activity at 128 $\mathrm{mg} / \mathrm{ml}$. By comparison, the MIC of tetracycline to MRSA Sp6

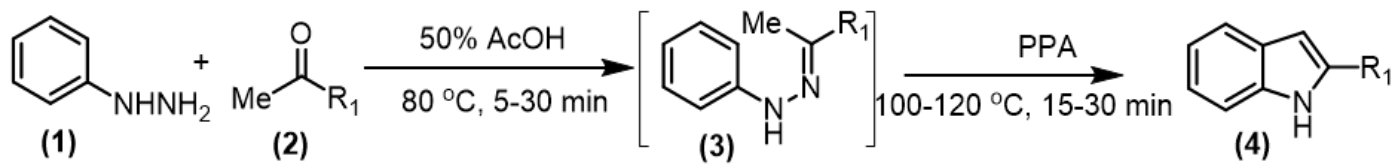

Scheme 1. Synthesis of 2-substituted phenyl- $1 H$ indole derivatives.<smiles>Nc1ccc(-c2cc3ccccc3[nH]2)cc1</smiles>

$(4 \mathrm{~m})$<smiles>[R]C(=O)Nc1ccc(-c2cc3ccccc3[nH]2)cc1</smiles>

6a; $\mathrm{R}_{2}=\mathrm{CH}_{3} \quad(53 \%)$

6b; $\mathrm{R}_{2}=\mathrm{CH}_{2} \mathrm{CH}_{3}(62 \%)$

6c; $\mathrm{R}_{2}=\left(\mathrm{CH}_{2}\right)_{6} \mathrm{CH}_{3} \quad(44 \%)$

6d; $\mathrm{R}_{2}=\mathrm{Ph}(90 \%)$

$6 \mathrm{e} ; \mathrm{R}_{2}=2-\mathrm{NO}_{2} \mathrm{Ph}(88 \%)$

6f; $\mathrm{R}_{2}=4-\mathrm{NO}_{2} \mathrm{Ph}(47 \%)$

Scheme 2. Preparation of N-substituted aminobenzene derivatives $(\mathbf{6 a}-\mathbf{f})$. 
Table 2. In vitro antimicrobial activities of compounds against the tested bacteria.

\begin{tabular}{|c|c|c|c|c|c|}
\hline \multirow{3}{*}{ Compound } & \multicolumn{5}{|c|}{$M I C(\mu g / m l)$} \\
\hline & \multicolumn{3}{|c|}{ Gram-positive } & \multicolumn{2}{|c|}{ Gram-negative } \\
\hline & S. aureus & B. subtilis & B. cereus & S. typhi & E. coli \\
\hline $4 a$ & na & na & na & $\mathrm{nt}$ & na \\
\hline $4 b$ & 250 & 125 & 62.5 & $\mathrm{nt}$ & 500 \\
\hline $4 c$ & $>500$ & 31 & $>500$ & $>500$ & $>500$ \\
\hline $4 d$ & $>500$ & 125 & $>500$ & $>500$ & $>500$ \\
\hline $4 e$ & 250 & $>500$ & $>500$ & $>500$ & 250 \\
\hline $4 f$ & $>500$ & 62 & $>500$ & $>500$ & $>500$ \\
\hline $4 g$ & 31 & 250 & $>500$ & $>500$ & $>500$ \\
\hline $4 h$ & $>500$ & $>500$ & $>500$ & $>500$ & $>500$ \\
\hline $4 i$ & 31 & 62 & $>500$ & 500 & 250 \\
\hline $4 j$ & 125 & $>500$ & $>500$ & 15.6 & $>500$ \\
\hline $4 k$ & 62 & 125 & 125 & 250 & 125 \\
\hline 41 & $>500$ & 250 & 500 & $>500$ & $>500$ \\
\hline $4 m$ & $>500$ & 15.6 & $>500$ & 15.6 & $>500$ \\
\hline $6 a$ & $>500$ & 250 & $>500$ & $>500$ & $>500$ \\
\hline $6 b$ & $>500$ & $>500$ & $>500$ & $>500$ & $>500$ \\
\hline $6 c$ & $>500$ & $>500$ & $>500$ & $>500$ & $>500$ \\
\hline 6d & $>500$ & $>500$ & $>500$ & $>500$ & $>500$ \\
\hline $6 e$ & $>500$ & $>500$ & $>500$ & $>500$ & $>500$ \\
\hline $6 f$ & $>500$ & $>500$ & $>500$ & $>500$ & $>500$ \\
\hline Kanamycin & 7.8 & $£ 1.9$ & 7.8 & 7.8 & 7.8 \\
\hline Chloramphenicol & 31.25 & $£ 1.9$ & 3.9 & $£ 1.9$ & $£ 1.9$ \\
\hline
\end{tabular}

was $256 \mathrm{mg} / \mathrm{ml}$. Nevertheless, the MIC of tetracycline decreased notably when the 2-arylindole derivatives were added. This result demonstrated that the sensitivity of MRSA Sp6 to tetracycline improved. Each experiment was repeated three times (Table 3). 4g, $\mathbf{4 j}$, and $\mathbf{4 k}$ elicited significant synergistic effects with tetracycline against MRSA Sp6. Thus, the MIC of tetracycline could be reduced from 256 to $32 \mathrm{mg} / \mathrm{ml}$ when it was combined with $\mathbf{4 g}, \mathbf{4 j}$, and $4 \mathbf{k}$ at a concentration of $16 \mathrm{mg} / \mathrm{ml}$.

\section{Molecular docking}

Molecular docking was conducted to explore the protein-substrate interactions and binding positions of the active synergistic compounds, namely, $\mathbf{4 g - k}$ and $\mathbf{4 m}$, in the active site of NorA from $S$. aureus. These compounds were docked into the active site of the $S$. aureus NorA model prepared with the EmrD efflux pump as a template. Their molecular docking
Table 3. Synergistic effect of 2-arylindoles with tetracyclin against MRSA $^{\mathrm{a}}$.

\begin{tabular}{cccccc}
\hline \multirow{2}{*}{ Substrates } & \multicolumn{5}{c}{ Tetracyclin $(\mathbf{m g} / \mathbf{m l})$} \\
& $\mathbf{0}$ & $\mathbf{1 6}$ & $\mathbf{3 2}$ & $\mathbf{6 4}$ & $\mathbf{1 2 8}$ \\
\hline $\mathbf{4 a}$ & $>128$ & $>128$ & $>128$ & 64 & 32 \\
$\mathbf{4 c}$ & $>128$ & 64 & 64 & 32 & 16 \\
$\mathbf{4 e}$ & $>128$ & $>128$ & $>128$ & 128 & 64 \\
$\mathbf{4 f}$ & $>128$ & 128 & 64 & 32 & 16 \\
$\mathbf{4 g}$ & $>128$ & 32 & 16 & 16 & 16 \\
$\mathbf{4 h}$ & $>128$ & 64 & 32 & 16 & 16 \\
$\mathbf{4 i}$ & $>128$ & 64 & 32 & 16 & 16 \\
$\mathbf{4 j}$ & $>128$ & 32 & 16 & 16 & 16 \\
$\mathbf{4 k}$ & $>128$ & 32 & 16 & 16 & 16 \\
$\mathbf{4 l}$ & $>128$ & 32 & 32 & 16 & 16 \\
$\mathbf{4} \mathbf{m}$ & $>128$ & 64 & 64 & 64 & 32 \\
$\mathbf{6 a}$ & $>128$ & $>128$ & $>128$ & $>128$ & $>128$ \\
$\mathbf{6 b}$ & $>128$ & $>128$ & $>128$ & $>128$ & $>128$ \\
$\mathbf{6 c}$ & $>128$ & $>128$ & $>128$ & $>128$ & $>128$ \\
$\mathbf{6 d}$ & $>128$ & $>128$ & $>128$ & 128 & 64 \\
$\mathbf{6 e}$ & $>128$ & $>128$ & 128 & 64 & 64 \\
$\mathbf{6 f}$ & $>128$ & $>128$ & $>128$ & 128 & 64 \\
\hline
\end{tabular}

${ }^{a}$ MRSA, Methicillin-resistant $S$. aureus (clinical isolate);: genotype: $m e c A$ positive, and tet $K$-positive.

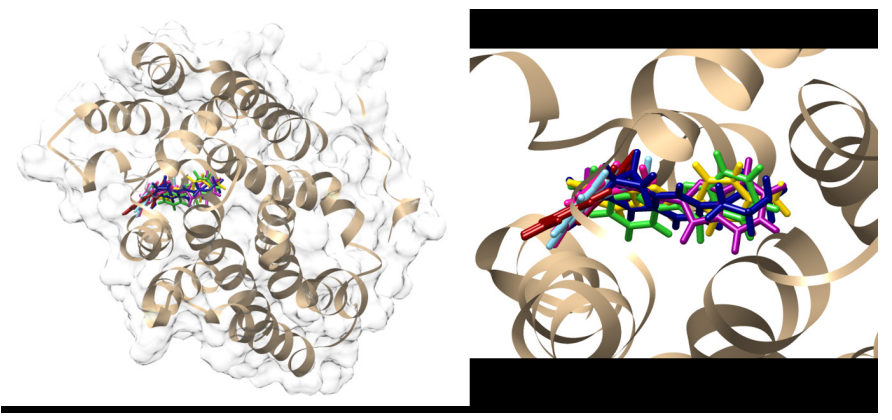

Figure 2. Comparison of the binding positions of $4 \mathrm{~g}$ (pink), $4 \mathrm{~h}$ (light blue), $4 \mathrm{i}$ (purple), $4 \mathbf{j}$ (green), $4 \mathbf{k}$ (red), $4 \mathrm{~m}$ (yellow), and ciprofloxacin (deep blue) in the cavity of the S. aureus NorA model prepared using the EmrD efflux pump (PDB ID: $2 \mathrm{GFP}$ ) as a template.

results were compared with those of the NorA substrate ciprofloxacin. The findings revealed that $\mathbf{4 g - k}$ and $\mathbf{4} \mathbf{~ m}$ bound to the cavity of the NorA efflux pump in a position similar to

Table 4. Binding energy $(\mathrm{kcal} / \mathrm{mol})$ and residues involved in the interaction of $\mathbf{4 g}-\mathbf{k}, \mathbf{4 m}$, and ciprofloxacin to the NorA efflux pump model.

\begin{tabular}{cccc}
\hline Compounds & $\begin{array}{c}\text { Binding energy } \\
\text { (kcal/mol) }\end{array}$ & Hydrogen bond & $\begin{array}{c}\text { Residue implicated in the interaction } \\
\text { Hydrophobic }\end{array}$ \\
\hline $\mathbf{4 g}$ & -90.37 & Glu222, Ile240 & Ile240, Ala243, Ala289 \\
$\mathbf{4 h}$ & -91.95 & Glu222, Trp293 & Ile240, Ala243, Ala289 \\
$\mathbf{4 i}$ & -85.07 & Asn290, Asp291 & Ile23, Val44, Ile240 \\
$\mathbf{4}$ & -91.73 & Asn290, Asp291, Trp293 & Val44, Phe47, Ile240, Ala243, Ile244, \\
$\mathbf{4}$ & -92.00 & Ser226 & Tlr292, \\
$\mathbf{4 k}$ & -91.46 & Phe47 & Va144, Phe47, Ile240, Ala243, Tyr292 \\
$\mathbf{4 m}$ & -99.64 & Asn290, Asp291, Trp293 & Tyr292, Ile240 \\
\hline
\end{tabular}




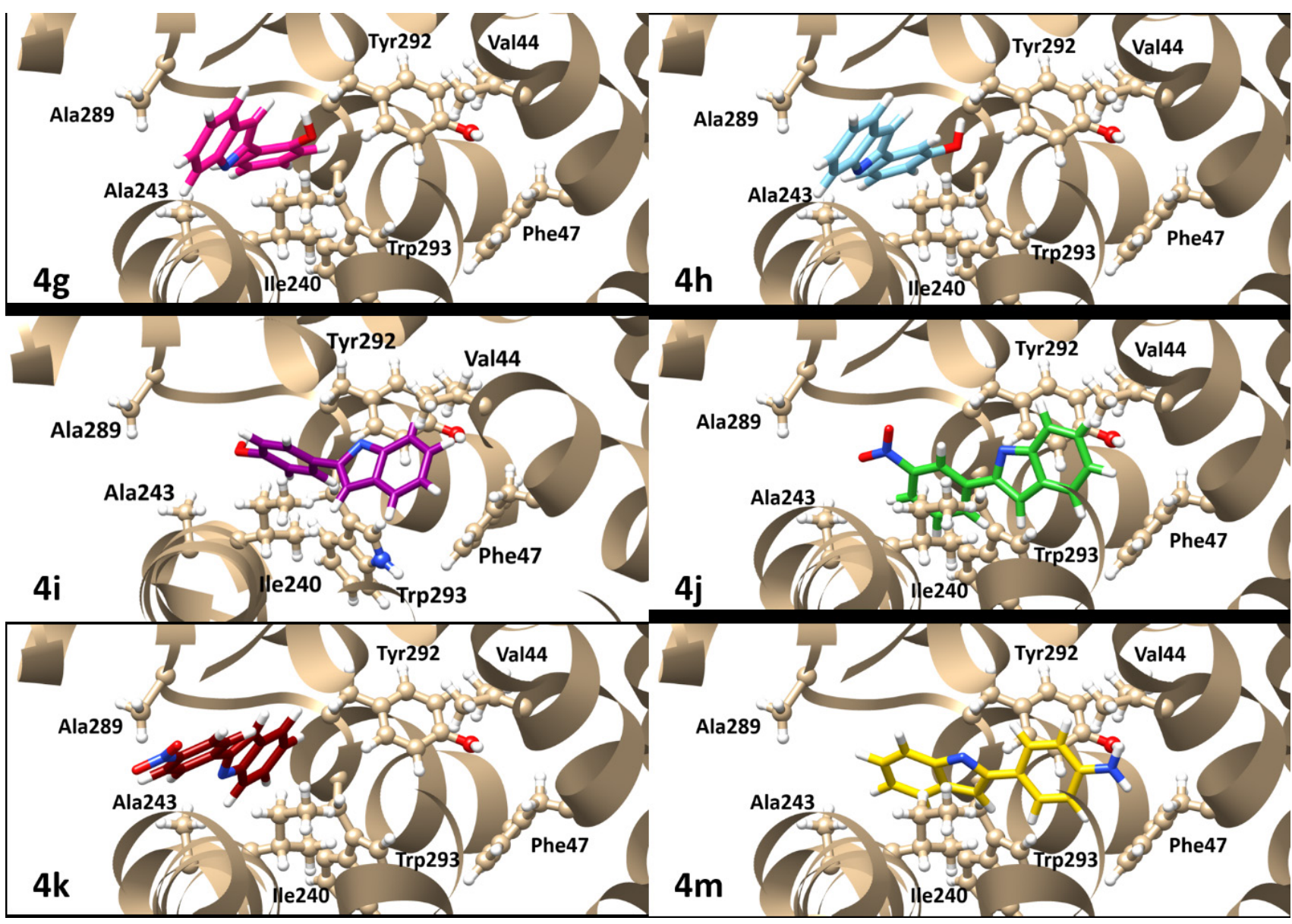

Figure 3. Binding positions of $\mathbf{4 g}-\mathbf{k}$ and $\mathbf{4 m}$ in the cavity of the NorA efflux pump model.

Table 5. ADMET properties predicted for compounds $\mathbf{4 a -} \mathbf{m}$ and $\mathbf{6 a}-\mathbf{f}$.

\begin{tabular}{|c|c|c|c|c|c|c|c|c|c|}
\hline & \multirow{2}{*}{ BBB } & \multirow{2}{*}{ HIA } & \multirow{2}{*}{ CPM } & \multirow{2}{*}{$\begin{array}{l}\text { CYP_2C19 } \\
\text { Inhibition }\end{array}$} & \multirow{2}{*}{$\begin{array}{l}\text { CYP_2C9 } \\
\text { Inhibition }\end{array}$} & \multicolumn{2}{|c|}{ CYP_2D6 } & \multicolumn{2}{|c|}{ CYP_3A4 } \\
\hline & & & & & & Inhibition & Substrate & Inhibition & Substrate \\
\hline $4 a$ & 1.50 & 100.00 & 22.28 & Inhibitor & Inhibitor & Non & Non & Inhibitor & Non \\
\hline $4 c$ & 13.68 & 100.00 & 22.37 & Inhibitor & Inhibitor & Non & Weakly & Inhibitor & Non \\
\hline $4 e$ & 10.90 & 95.09 & 31.32 & Inhibitor & Inhibitor & Non & Non & Inhibitor & Non \\
\hline $4 f$ & 11.58 & 95.09 & 31.32 & Inhibitor & Inhibitor & Non & Non & Inhibitor & Non \\
\hline $4 \mathrm{~g}$ & 8.65 & 91.94 & 38.13 & Inhibitor & Inhibitor & Non & Non & Inhibitor & Non \\
\hline $4 \mathrm{~h}$ & 8.65 & 91.94 & 3.53 & Inhibitor & Inhibitor & Non & Non & Inhibitor & Non \\
\hline $4 \mathbf{i}$ & 8.65 & 91.94 & 3.53 & Inhibitor & Inhibitor & Non & Non & Inhibitor & Non \\
\hline $4 \mathbf{j}$ & 3.56 & 91.70 & 3.61 & Inhibitor & Inhibitor & Non & Non & Inhibitor & Weakly \\
\hline $4 k$ & 3.29 & 91.70 & 3.61 & Inhibitor & Inhibitor & Non & Non & Inhibitor & Non \\
\hline 41 & 14.23 & 100.00 & 29.24 & Inhibitor & Inhibitor & Non & Non & Inhibitor & Non \\
\hline $4 m$ & 5.95 & 91.90 & 6.53 & Inhibitor & Inhibitor & Non & Non & Inhibitor & Non \\
\hline $6 a$ & 4.25 & 91.82 & 39.54 & Non & Non & Non & Non & Non & Non \\
\hline $6 b$ & 6.43 & 92.04 & 44.68 & Non & Non & Non & Non & Non & Non \\
\hline $6 c$ & 14.55 & 93.09 & 54.98 & Non & Non & Inhibitor & Non & Non & Non \\
\hline 6d & 9.30 & 93.35 & 48.59 & Non & Non & Non & Non & Non & Non \\
\hline $6 e$ & 0.70 & 92.05 & 18.47 & Non & Non & Non & Non & Non & Weakly \\
\hline $6 f$ & 1.59 & 92.05 & 19.58 & Non & Non & Non & Non & Non & Weakly \\
\hline
\end{tabular}

BBB, Blood-brain barrier; HIA, Human intestinal absorption; CPM, Caco-2-cells permeability. 
that of ciprofloxacin (Fig. 2). The binding energies of $\mathbf{4 g}-\mathbf{k}$ and $4 \mathrm{~m}$ were -92.00 to $-85.07 \mathrm{kcal} / \mathrm{mol}$ (Table 4 ), which were slightly higher than that of ciprofloxacin (-99.64 kcal/ $\mathrm{mol})$. Moreover, in the active site of the NorA efflux pump, $\mathbf{4 g}$, 4h, and $4 \mathbf{k}$ interacted with key amino acid residues, namely, Ile240, Ala243, and Ala289, respectively, while $\mathbf{4 i}, \mathbf{4 j}$, and $\mathbf{4 m}$ interacted with Val44, Phe47, and Ile240, respectively (Fig. $3)$. Therefore, the aromatic moieties of $\mathbf{4 g}-\mathbf{k}$ and $\mathbf{4 m}$ played important roles in the binding of these compounds to the hydrophobic core of the NorA efflux pump.

\section{Prediction of ADMET by computational analysis}

The ADMET properties of the synthesized compounds were presented in Table 5. This result suggested that most of the compounds were through to cross BBB permeation. For in vitro Caco-2 cell permeability, most of the compounds except $\mathbf{4 h}-\mathbf{k}$ and $\mathbf{4 m}$ have high permeability and are easy to absorb. All of the compounds showed \% HIA in the range of $91.70 \%$ $100.00 \%$ which were considered to be very well absorbed in the gastrointestinal tract. The toxicity predictions were considered of the cytochrome P450s (CYP), an important enzyme system for drug metabolism that influences clearance rates, toxicity, and interactions with coadministered drugs. All compounds were predicted to be not substrates and not inhibitors for four major CYP isoforms, except for compounds $\mathbf{4 a}-\mathbf{m}$, which were predicted to be CYP2C19, CYP2C9, and CYP3A4 inhibitors. This suggested that these compounds may be metabolized in the liver which may have hepatotoxicity.

\section{CONCLUSION}

2-Arylindole derivatives were synthesized, and their antibacterial activity and synergistic effects on multidrugresistant $S$. aureus were evaluated. The results showed that $4 \mathbf{j}$ combined with tetracycline showed a potent antibacterial activity against $S$. typhi and exhibited a synergistic effect against MRSA Sp6 strain. Furthermore, $4 \mathbf{~ m}$ displayed the most potent antibacterial activity against $B$. subtilis, whereas $\mathbf{4 j}$ was the most potent against $S$. typhi. All the synthesized compounds improved the sensitivity of MRSA Sp6 to tetracycline. Molecular docking studies on $\mathbf{4 g}, \mathbf{4 h}$, and $\mathbf{4 k}$ revealed that interaction with key amino acids occurred at the active site of the NorA efflux pump. However, the toxicity study by ADMET was predicted as hepatotoxicity. Therefore, the 2-arylindole derivatives could be considered as an interesting scaffold and further development of the 2-arylindole derivative with no toxicity might be the leading compounds that could potentiate the activity of commercially available drugs against multidrugresistant bacteria.

\section{ACKNOWLEDGEMENTS}

We gratefully acknowledge the Department of Chemistry and the Department of Microbiology, Faculty of Science, Silpakorn University, for the financial support and antibacterial and synergistic effect assay. We also thank the Chulabhorn Research Institute for the measurements of the HR-ESI mass spectroscopy investigation.

\section{AUTHOR CONTRIBUTIONS}

All authors made substantial contributions to conception and design, acquisition of data, or analysis and interpretation of data; took part in drafting the article or revising it critically for important intellectual content; agreed to submit the paper to the current journal; gave final approval of the version to be published; and agree to be accountable for all aspects of the work. All the authors are eligible to be an author as per the international committee of medical journal editors requirements/guidelines.

\section{CONFLICTS OF INTEREST}

The authors report no financial or any other conflicts of interest in this work.

\section{FUNDING}

There is no funding to report.

\section{ETHICAL APPROVAL}

This study does not involve experiments on animals or human subjects.

\section{PUBLISHER'S NOTE}

This journal remains neutral with regard to jurisdictional claims in published institutional affiliation.

\section{REFERENCES}

Ackermann L, Barfuesser S, Potukuchia H K. Coppercatalyzed $\mathrm{N}$-arylation/hydroamin(d)ation domino synthesis of indoles and its application to the preparation of a Chek1/KDR kinase inhibitor pharmacophore. Adv Synth Catal, 2009; 351:1064-72; http://doi. org/10.1002/adsc.200900004

Bremner JB, Ambrus JI, Kelso MJ, Ball AR, Casadei G, Lewis K. Structure-activity relationships of 2-aryl-1H-indole inhibitors of the NorA efflux pump in Staphylococcus aureus. Bioorg Med Chem Lett, 2008; 18:4294-7; http://doi.org/10.1016/j.bmcl.2008.06.093

Brvar M, Perdih A, Renko M, Anderluh G, Turk D, Solmajer T. Structure-based discovery of substituted $4,5^{\prime}$-bithiazoles as novel DNA gyrase inhibitors. J Med Chem, 2012; 55:6413-26; http://doi.org/10.1021/ jm300395d

Cho C, Park I, Suh S, Lim B. Aryl hydrazide beyond as surrogate of aryl hydrazine in the Fischer indolization: the synthesis of $N$-Cbz-indoles, $N$-Cbz-carbazoles, and $N, N^{\prime}$-Bis-Cbz-pyrrolo[2,3-f]indoles. Org Lett, 2009; 11:5454-6; http://doi.org/10.1021/o1902250x

CLSI. Performance standards for antimicrobial susceptibility testing: 24th informational supplement, CLSI document M100-S24 Clinical and Laboratory Standards Institute, Wayne, PA, 2014.

Cossío FP, Vara Y, Aldaba E, Arrieta A, Pizarro JL, Arriortua MI. Regiochemistry of the microwave-assisted reaction between aromatic amines and $\alpha$-bromoketones to yield substituted $1 H$-indoles. Org Biomol Chem, 2008; 6:1763-72; http://doi.org/10.1039/B719641E

Dai WM, Guo DS, Sun LP. Chemistry of aminophenols. Part 1: Remarkable additive effect on Sonogashira cross-coupling of 2-carboxamidoaryl triflates and application to novel synthesis of indoles. Tetrahedron Lett, 2001; 42:5275-8; http://doi.org/10.1016/S00404039(01)00965-0

Deschenes RJ, Lin H, Ault AD, Fassler JS. Antifungal properties and target evaluation of three putative bacterial histidine kinase inhibitors. Antimicrob Agents Chemother, 1999; 43(7):1700-3. http://doi.org/10.1128/ AAC.43.7.1700.

Ezquerra J, Pedregal C, Lamas C, Barluenga J, Pérez M, GarcíaMartín MA, González JM. Efficient reagents for the synthesis of 5-, 7-, and 
5,7-substituted indoles starting from aromatic amines: scope and limitations. J Org Chem, 1996; 61:5804-12; http://doi.org/10.1021/jo952119+

Fuher W, Gschwend HW. Ortho functionalization of aromatic amines: ortho lithiation of N-pivaloylanilines. J Org Chem, 1979; 44(7):1133-6; http://doi.org/10.1021/jo01321a023

Heck RF, Terpko MO. Rearrangement in the palladium-catalyzed cyclization of alpha.-substituted N-acryloyl-o-bromoanilines. J Chem Soc, 1979; 101(18):5281-3; http://doi.org/10.1021/ja00512a028

Hibino S, Chosi T. Simple indole alkaloids and those with a nonrearranged monoterpenoid unit. Nat Prod Rep, 2002; 19:148-80; http:// doi.org/10.1039/B007740M

Hong BC, Jiang Y, Chang Y, Lee S. Synthesis and cytotoxicity studies of cyclohepta[$[\beta]$ indoles, benzo[6,7]cyclohepta[1,2- $\beta]$ indoles, indeno[1,2- $\beta]$ indoles, and benzo[ $\alpha]$ carbazoles. J Chin Chem Soc, 2006; 53:647-62; http://doi.org/10.1002/jccs.200600086

Hughes DL. Progress in the Fischer indole reaction. N J Chem, 1993; 6:607-32; http://doi.org/10.1080/00304949309356257

Imanishi T, Miyashita K, Kondoh K, Tsuchiya K, Miyabe $\mathrm{H}$. Novel indole-ring formation by thermolysis of 2-(N-acylamino)benzylphosphonium salts. Effective synthesis of 2-trifluoromethylindoles. J Chem Soc Perkin Trans, 1996; 1:1261-8; http://doi.org/10.1039/ P19960001261

Kim BM, Kwon J, Chung J, Byun S. Efficient synthesis of indole derivatives via tandem cyclization catalyzed by magnetically recoverable palladium/magnetite $\left(\mathrm{Pd}-\mathrm{Fe}_{3} \mathrm{O}_{4}\right.$ ) nanocrystals. Asian J Org Chem, 2016; 5:470 -6; http://doi.org/10.1002/ajoc.201500536

Kuduk SD, Chang RK, Wai JM, Di Marco CN, Cofre V, DiPardo RM, Cook SP, Cato MJ, Jovanovska A, Urban MO, Leitl M, Spencer RH, Kane SA, Hartman GD, Bilodeau MT. Amidine derived inhibitors of acid-sensing ion channel-3 (ASIC3). Bioorg Med Chem Lett, 2009; 19(15):4059-63; http://doi.org/10.1016/j.bmcl.2009.03.029

Lal S, Snape TJ. 2-arylindoles: a privileged molecular scaffold with potent, broad-ranging pharmacological activity. Curr Med Chem, 2012; 19:4828-37; http://doi.org/10.2174/092986712803341449

Larock RC, Yum EK Synthesis of indoles via palladiumcatalyzed heteroannulation of internal alkynes. J Am Chem Soc, 1991; 113(17):6689-90; http://doi.org/10.1021/ja00017a059

Markham PN, Westhaus E, Klyachko K, Johnson EM, Neyfakh AA. Multiple novel inhibitors of the nora multidrug transporter of Staphylococcus aureus. Antimicrob Agents Chemother, 1999; 43(10):24048; http://doi.org/10.1128/aac.43.10.2404

Naik M, Ghorpade S, Jena LK, Gorai G, Narayan A, Guptha S, Sharma S, Dinesh N, Kaur P, Nandishaiah R, Bhat J, Balakrishnan G, Humnabadkar V, Ramachandran V, Naviri L K, Khadtare P, Panda M, Iyer PS, Chatterji M. 2-phenylindole and arylsulphonamide: novel scaffolds bactericidal against Mycobacterium tuberculosis. ACS Med Chem Lett, 2014; 5(9):1005-9; http://doi.org/10.1021/ml5001933

Nakazaki M, Yamamoto K. Direct synthesis of indole by the fischer indole synthesis. J Org Chem, 1976; 41(10):1877; http://doi. org/10.1021/jo00872a045

National Committee for Clinical Laboratory Standards. Methods for dilution antimicrobial susceptibility tests for bacteria that grow aerobically; approved standard. NCCLS Document M7-A4, Villanova, PA, 1997.

Reygaert WC. An overview of the antimicrobial resistance mechanisms of bacteria. Microbiology, 2018; 4(3):482-501; http://doi. org/10.3934/microbiol.2018.3.482
Rudisill DE, Stille JK. Palladium-catalyzed synthesis of 2-substituted indoles. J Org Chem, 1989; 54:5856-66; http://doi. org/10.1021/jo00286a014

Samosorn S, Bremner JB, Ball A, Lewis K. Synthesis of functionalised 2-aryl-5-nitro- $1 \mathrm{H}$-indoles and their activity as bacterial NorA efflux pump inhibitors. Bioorg Med Chem, 2006; 14 (3):857-65; http://doi. org/10.1016/j.bmc.2005.09.019

Shi J, Yang SD, Sun CL, Fang Z, Li BJ, Li YZ. Palladiumcatalyzed arylation of (Het)arenes with arylboronic acids. Synfacts, 2008; 5:0514; http://doi.org/10.1055/s-2008-1072518

Snape T, Prabhu S, Akbar Z, Harris F, Karakoula K, Lea R, Rowther F, Warr T. Preliminary biological evaluation and mechanism of action studies of selected 2-arylindoles against glioblastoma. Bioorg Med Chem, 2013; 21:1918-24; http://doi.org/org/10.1016/j.bmc.2013.01.032

Taechowisan T, Mungchukeatsakul N, Phutdhawong WS. Antimicrobial resistance pattern of Staphylococcus aureus strains isolates from clinical and hospital environment specimens and their correleation with PCR-based approaches. Res J Microbiol, 2018; 13:100-18; http://doi. org/10.3923/jm.2018.100.118

Tsuchimoto T, Matsubayashi H, Kaneko M, Shirakawa E, Kawakami Y. Easy Access to aryl-and heteroarylannulated[a]carbazoles by the indium-catalyzed reaction of 2-arylindoles with propargyl ethers. Angew Chem Int Ed, 2005; 44:1336-40; http://doi.org/10.1002/anie.200462280

Taylor EC, Katz AH, Salgado-Zamora H, McKillop AM. Thallium in organic synthesis. 68. A convenient synthesis of 2-phenylindoles from anilides. Tetrahedron Lett, 1985; 26:5963-6; http://doi.org/10.1016/ S0040-4039(00)98272-8

Westwell AD, Suzen S, Coban T, Karaaslan C, Kadri H. Synthesis and antioxidant properties of substituted 2-phenyl-1H-indoles. Bioorg Med Chem Lett, 2013; 23:2671-4; http://doi.org/org/10.1016/j. bmcl.2013.02.090

Williams JD, Nguyen ST, Gu S, Ding X, Butler MM, Tashjian TF, Opperman TJ, Panchal RG, Bavari S, Peet NP, Moir DT, Terry L Bowlin TL. Potent and broad-spectrum antibacterial activity of indole-based bisamidine antibiotics: synthesis and SAR of novel analogs of MBX 1066 and MBX 1090. Bioorg Med Chem, 2013; 21(24):7790-806; http://doi. org/10.1016/j.bmc.2013.10.014

Zárate SG, Morales P, Świderek K, Bolanos-Garcia VM, Bastida A. A molecular modeling approach to identify novel inhibitors of the major facilitator superfamily of efflux pump transporters. Antibiotics, 2019; 8(1):25; http://doi.org/10.3390/antibiotics8010025

\section{How to cite this article:}

Vicharn S, Sirirak J, Phutdhawong W, Taechowisan T, Phutdhawong WS. Synthesis of 2-substituted indoles and evaluation of their antibacterial activity and inhibitory effects on the efflux pump of methicillin-resistant Staphylococcus aureus. J Appl Pharm Sci, 2022; 12(01):084-093. 